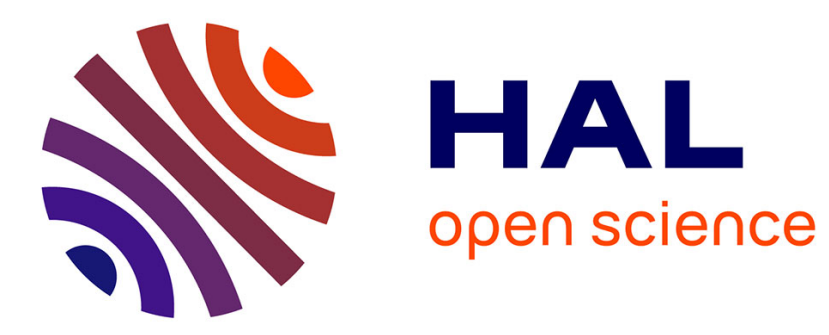

\title{
Global stability of swept flow around a parabolic body: The neutral curve
}

Christoph J. Mack, Peter J. Schmid

\section{To cite this version:}

Christoph J. Mack, Peter J. Schmid. Global stability of swept flow around a parabolic body: The neutral curve. Journal of Fluid Mechanics, 2011, 678, pp.589-599. 10.1017/jfm.2011.158 . hal00997980

\section{HAL Id: hal-00997980 \\ https://hal-polytechnique.archives-ouvertes.fr/hal-00997980}

Submitted on 5 Jun 2014

HAL is a multi-disciplinary open access archive for the deposit and dissemination of scientific research documents, whether they are published or not. The documents may come from teaching and research institutions in France or abroad, or from public or private research centers.
L'archive ouverte pluridisciplinaire HAL, est destinée au dépôt et à la diffusion de documents scientifiques de niveau recherche, publiés ou non, émanant des établissements d'enseignement et de recherche français ou étrangers, des laboratoires publics ou privés. 


\title{
Global stability of swept flow around a parabolic body: the neutral curve
}

\author{
CHRISTOPH J. MACK ${ }^{1,2}$ AND PETER J. SCHMID ${ }^{1} \dagger$ \\ ${ }^{1}$ Laboratoire d'Hydrodynamique (LadHyX), CNRS-École Polytechnique, F-91128 Palaiseau, France \\ ${ }^{2}$ Department of Numerical Mathematics, Universität der Bundeswehr (UniBw), \\ D-85577 Munich, Germany
}

(Received 17 September 2010; revised 22 March 2011; accepted 28 March 2011; first published online 12 May 2011)

The onset of transition in the leading-edge region of a swept blunt body depends crucially on the stability characteristics of the flow. Modelling this flow configuration by swept compressible flow around a parabolic body, a global approach is taken to extract pertinent stability information via a DNS-based iterative eigenvalue solver. Global modes combining features from boundary-layer and acoustic instabilities are presented. A parameter study, varying the spanwise disturbance wavenumber and the sweep Reynolds number, showed the existence of unstable boundary-layer and acoustic modes. The corresponding neutral curve displays two overlapping regions of exponential growth and two critical Reynolds numbers, one for boundary-layer instabilities and one for acoustic instabilities. The employed global approach establishes a first neutral curve, delineating stable from unstable parameter configurations, for the complex flow about a swept parabolic body with corresponding implications for swept leading-edge flow.

Key words: boundary layer stability, compressible boundary layers

\section{Introduction}

Since the early 1950s, the problem of transition in the leading-edge region of swept wings has been central to a great deal of investigations concerning the design of highperformance aircraft. This problem was first observed in early flight tests on swept wing aircraft, where Gray (1952) found that, beyond a critical free-stream velocity, the transition front moved towards the attachment line of the wing, a feature that could not be explained by invoking two-dimensional arguments. Furthermore, the employed flow visualization technique showed a series of closely spaced streaky structures almost aligned with the external streamlines of the flow. Subsequent investigations confirmed these streaky structures as a consequence of crossflow instabilities.

In an attempt to gather further information on leading-edge transition, Pfenninger conducted a series of flight tests on a swept X-21 wing in 1963. He observed that unexpectedly high suction rates had to be applied to eliminate the crossflow vortices and, therefore, to achieve laminar flow over the outer part of the wing. This strong suction was required particularly in the wing's leading-edge region which indicated that rather strong disturbances had to be present in the laminar boundary layer 
originating near the attachment line. Evaluating his database, Pfenninger (1965) was able to establish a criterion for the existence of spanwise contamination along the wing's leading edge in the presence of an initially turbulent attachment-line boundary layer emanating from the wing-body junction. He further realized that maintaining a full-chord laminar flow on an X-21 wing critically depended on the existence of an undisturbed laminar attachment-line boundary layer, thus stressing the importance of flow instabilities in the vicinity of the attachment line. Practical consequences of the above early findings are reflected in the proposition of geometric devices, introduced near the attachment line, to prevent the onset of instabilities and to extend the parameter regime for laminar flow near the leading edge (see Gaster 1965, 1967). Pfenninger's criterion is expressed in terms of a critical sweep Reynolds number of $\bar{R} \approx 245$, which corresponds to a Reynolds number of $R_{\theta} \approx 100$ (based on the momentum thickness), to avoid leading-edge contamination. Presently, this value still guides state-of-the-art design efforts for swept wings.

The observed prevalence of crossflow vortices in the experiments of, among others, Pfenninger (1965) and Poll (1979) led, in the beginning, to the hypothesis of a leading-edge transition caused by crossflow vortices; however, doubts remained as to their singular role. In wind-tunnel experiments on a swept wing configuration with a semi-circular leading edge, Poll (1979) observed that the flow is also susceptible to instabilities close to the attachment line. As a result, he established attachment-line instabilities as a viable alternative governing the stability of swept leading-edge flow. Summarizing his investigations on incompressible attachment-line flow, he confirmed Pfenninger's critical Reynolds number of $\bar{R} \approx 245$ for large-amplitude disturbances. In addition to that, Pfenninger reported a critical Reynolds number of $\bar{R}_{\text {crit }} \approx 570$ for small-amplitude perturbations. The discrepancy between these two critical Reynolds numbers suggests a subcritical nature of leading-edge transition, and an interplay between linear and nonlinear mechanisms is expected to play a considerable role in this parameter range (see e.g. Joslin 1996).

Over time, the early studies of incompressible or weakly compressible flows have been extended, and experimental investigations into the subsonic, supersonic and hypersonic flow regime have been undertaken by many scientists. A comprehensive summary of their results is given in Poll (1983) and Gaillard, Benard \& Alziary de Roquefort (1999). Adopting a reference temperature concept, a unique critical Reynolds number of $\bar{R}^{*} \approx 245$ has been determined for a finite-amplitude disturbance environment (Poll 1983). For infinitesimal perturbations a critical Reynolds number of $\bar{R}_{\text {crit }}^{*} \approx 650$ has been found (see e.g. Gaillard et al. 1999). In both cases, the critical values of the Reynolds number appear to be independent of the sweep Mach number $M a_{s}$ up to a value of $M a_{s} \approx 5$.

The experimental results as well as our desire to fully understand swept leadingedge flow have fuelled a substantial effort (see e.g. Hall, Malik \& Poll 1984; Hall \& Malik 1986; Spalart 1988; Joslin 1995; Lin \& Malik 1996; Semisynov et al. 2003) to investigate the flow behaviour governed by the two identified mechanisms, i.e. attachment-line instabilities and crossflow vortices. All these investigations, however, have been based on local flow models for either instability mechanism, which was a necessary simplification before classical tools of hydrodynamic stability theory could be applied. Despite remarkable theoretical and numerical efforts based on these local models, our understanding of the mechanisms underlying the perturbation dynamics in the subcritical range still remains incomplete, even though limited success, such as approximating the critical Reynolds number for attachment-line instabilities (Hall et al. 1984; Lin \& Malik 1996), has been achieved. 


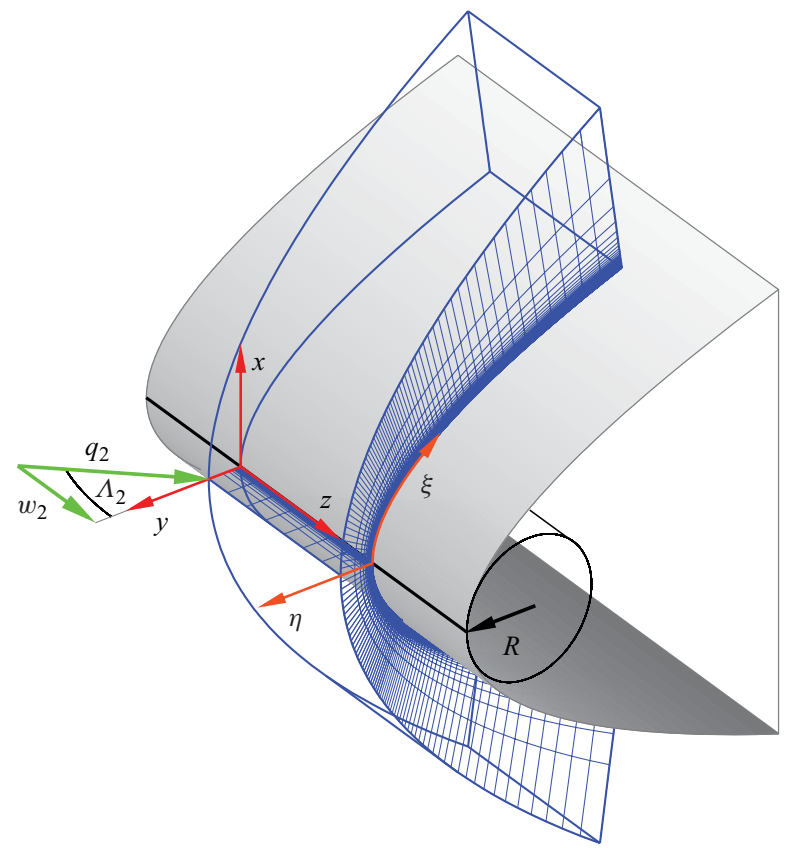

FIGURE 1. Sketch of the supersonic flow about a swept parabolic body.

The shortcomings of the local flow models have been discussed by a number of scientists, and the potential of a global stability approach based on a comprehensive flow model is unquestionable. Such an approach will yield a more complete understanding of swept leading-edge flow and help answer questions such as the connection between attachment-line instabilities and crossflow vortices. An approach of this type has already been alluded to by Lin \& Malik (1996), who stated that '... [b]y choosing a computational domain large enough in the [chordwise] direction to cover both the attachment-line instability and crossflow instability regions, and by using an appropriate spatial resolution, the two-dimensional eigenvalue approach can provide us with a means to explore this connection .... Based on such a global approach, this connection has been established by Mack, Schmid \& Sesterhenn (2008), and the same line of analysis will be pursued by more sophisticated tools in this article to explore the parameter dependence of swept flow around a parabolic body. In particular, a neutral stability curve for a global model of compressible swept leading-edge flow will be presented; to our knowledge, this curve represents - specifically - the first attempt to delineate stable and unstable parameter combinations for this type of flow and more generally - a neutral stability curve for a complex flow configuration described by global stability analysis.

\section{Flow model, governing parameters and numerical method}

Our model consists of supersonic flow about a swept parabolic body displayed (in grey) in figure 1 . The same figure introduces the used three-dimensional body-fitted grid (in blue) and two coordinate systems: a local $(x, y, z)$ Cartesian coordinate system (in red) and a local parabolic $(\xi, \eta, z)$ coordinate system (in orange). The attachment line (in black) coincides with the spanwise $z$-direction. The surface of the parabolic 
body is given by $y(x)=-1 /(2 R) x^{2}$, where $R$ denotes its leading-edge radius. We consider flow situations where the oncoming flow is supersonic $M_{1}=M_{\infty} \cos \Lambda>1$, with $M_{\infty}$ and $M_{1}$ respectively denoting the free-stream Mach number and its normal component, and $\Lambda$ denotes the free-stream angle. The computational domain is limited by a detached unsteady bow shock in the wall-normal direction, and this shock, assumed to be an infinitely thin, moving discontinuity, acts as the inflow boundary. The supersonic flow state upstream of the detached bow shock is given by the Mach number $M_{\infty}$, the free-stream angle $\Lambda$ as well as the pressure $p_{\infty}$ and temperature $T_{\infty}$. Via the Rankine-Hugoniot relations

$$
\left.\begin{array}{l}
u_{2}=u_{1}, \quad p_{2}=-\frac{\gamma-1}{\gamma+1} p_{1}+\frac{2}{\gamma+1} \rho_{1} v_{1}^{2}, \\
v_{2}=\frac{\gamma-1}{\gamma+1} v_{1}+\frac{2 \gamma}{\gamma+1} \frac{p_{1}}{\rho_{1} v_{1}}, \quad \rho_{2}=\frac{v_{1} \rho_{1}}{v_{2}}, \\
w_{2}=w_{1}, \quad s_{2}=C_{v} \ln \left(\frac{p_{2}}{\rho_{2}^{\gamma}}\right),
\end{array}\right\}
$$

where $u, v$ and $w$ respectively denote the (locally) tangential, normal and bi-normal velocity components and $p, \rho$ and $s$ denote respectively the pressure, density and entropy, the flow state downstream of the shock, indicated by the subscript 2 , is obtained. As a result, the incoming flow impinges on the body with a velocity $q_{2}$ and a sweep angle $\Lambda_{2}$ yielding a sweep velocity $w_{2}$ and a normal velocity $v_{2}$ (see figure 1 ).

This flow configuration is characterized by two distinct length scales, the leadingedge radius $R$ and a viscous length scale $\delta$, which describe the outer flow and the flow inside the boundary layer, respectively. We define the viscous length scale as

$$
\delta=\left(\frac{v_{r}}{S}\right)^{1 / 2} \quad \text { with } S=\left(\frac{\partial u}{\partial x}\right)_{w}=\frac{2 v_{2}}{R},
$$

where $S$ is the strain rate at the wall, evaluated at the attachment line. Its value is approximated using the potential solution of flow around a circular cylinder with radius $R$. On the basis of these two length scales, we define a sweep Reynolds number $R e_{s}$ and a leading-edge Reynolds number $R e_{R}$ as

$$
R e_{s}=\frac{w_{2} \delta}{v_{r}}, \quad R e_{R}=\frac{v_{2} R}{v_{r}},
$$

where $v_{r}$ denotes the kinematic viscosity evaluated at recovery temperature $T_{r}$ and stagnation pressure $p_{s}$. We further consider a supersonic sweep Mach number of $M a_{s}=w_{2} / c_{2}=1.25$ and assume an adiabatic wall, i.e. the ratio of the temperature $T_{w}$ at the wall and $T_{r}$ is $\theta_{w}=T_{w} / T_{r} \approx 1$.

It is instructive to demonstrate an explicit dependence of the sweep Reynolds number $R e_{s}$ on the sweep angle $\Lambda_{2}$ and the leading-edge radius $R$ as well as the leading-edge Reynolds number $R e_{R}$ according to

$$
R e_{s}=\left(\frac{q_{2} R}{v_{r}} \frac{\sin \Lambda_{2} \tan \Lambda_{2}}{2}\right)^{1 / 2}=\left(\frac{R e_{R}}{2}\right)^{1 / 2} \tan \Lambda_{2}=\frac{R}{2 \delta} \tan \Lambda_{2}
$$

The definition in (2.4) was used by Poll (1979) for incompressible flow over a swept cylinder. For compressible flow it was extended using a reference temperature concept (see Poll 1984) where the kinematic viscosity $v^{*}$ in the sweep Reynolds number $\bar{R}^{*}$ is evaluated at a reference temperature $T^{*}$ given by $T^{*}=T_{e}+0.1\left(T_{w}-T_{e}\right)+0.6\left(T_{r}-T_{e}\right)$; the temperature $T_{e}$ is computed at the boundary-layer edge. 
The governing compressible Navier-Stokes equations are solved on a curvilinear, moving and body-fitted mesh using high-order compact finite-difference schemes and high-order time stepping. Shock-fitting techniques, a characteristic-type formulation and standard non-reflecting outflow boundary conditions are used, and further details about the direct numerical simulation (DNS) are given by Mack \& Schmid (2010a). To properly resolve small-scale features inside the boundary layer as well as near the attachment line, a highly non-uniform grid distribution is employed, as indicated in figure 1.

\section{Global stability analysis}

It should be evident that stability studies of flow about a blunt body such as a swept wing no longer allow the standard simplifying assumptions of multiple homogeneous coordinate directions or a low-Mach-number approximation. Rather, a global approach has to be adopted to extract pertinent stability information about the flow. Such an approach has already been amply motivated in the Introduction, where we concluded that further advances in our understanding of leading-edge contamination critically rely on the formulation of a global stability problem.

We therefore assume a three-dimensional perturbation field, which in our case consists of the pressure, velocity components and entropy, i.e. $\phi^{\prime}=\left(p^{\prime}, u^{\prime}, v^{\prime}, w^{\prime}, s^{\prime}\right)^{\mathrm{T}}$, superimposed on a steady base flow $\phi_{0}$ according to

$$
\phi(x, y, z, t)=\phi_{0}(x, y, z)+\epsilon \phi^{\prime}(x, y, z, t), \quad \epsilon \ll 1 .
$$

The base flow $\phi_{0}$ is computed as a steady solution of the nonlinear compressible Navier-Stokes equations by time marching the DNS code.

Despite the complexity of our flow model, we can still take advantage of the homogeneous spanwise $z$-direction in the form of a Fourier transform in $z$ introducing a spanwise wavenumber $\beta$. Physically, this corresponds to a swept parabolic body of infinite span. Assuming exponential behaviour in time, we take the perturbation in (3.1) in the form of travelling waves according to

$$
\phi^{\prime}(x, y, z, t)=\widetilde{\phi}(x, y) \mathrm{e}^{\mathrm{i}(\beta z-\omega t)} .
$$

In this expression, $\widetilde{\phi}(x, y)$ denotes the complex amplitude of the disturbance, and its temporal long-term evolution is given by $\omega$ whose real part $\omega_{r}$ describes the frequency and imaginary part $\omega_{i}$ represents the corresponding growth rate. The complex frequency $\omega$ has been non-dimensionalized by the strain rate $2 v_{2} / R$; see (2.2).

Characteristic information about the stability behaviour of compressible flow about a swept parabolic body is contained in the global modes $\widetilde{\phi}(x, y)$, which present the spatial shape of the instabilities, and the corresponding global eigenvalues $\omega$, which describe the associated temporal dynamics. This information is extracted by applying a DNS-based iterative global stability solver which consists of the implicitly restarted Arnoldi method (see Lehoucq, Sorensen \& Yang 1998) combined with a conformal map (Cayley transformation) of the complex eigenvalue plane. By implementing a Jacobian-free framework, flow fields computed by the DNS provide the required input for the Arnoldi method, and a shift parameter $\sigma$ in the Cayley transformation allows us to manipulate the computed flow fields and direct the convergence of the Arnoldi method towards specific global modes. In this manner, we are able to scan the physically relevant regions of the full global stability spectrum. The computed global spectrum has been confirmed to be independent of numerical parameters and approximations, such as resolution, grid distribution and location of the outflow 


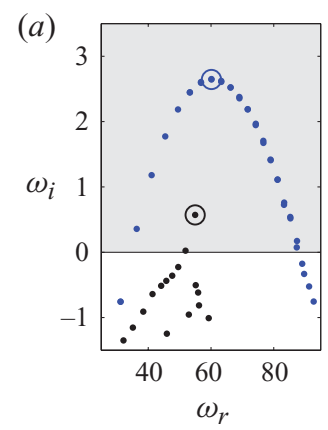

(b)

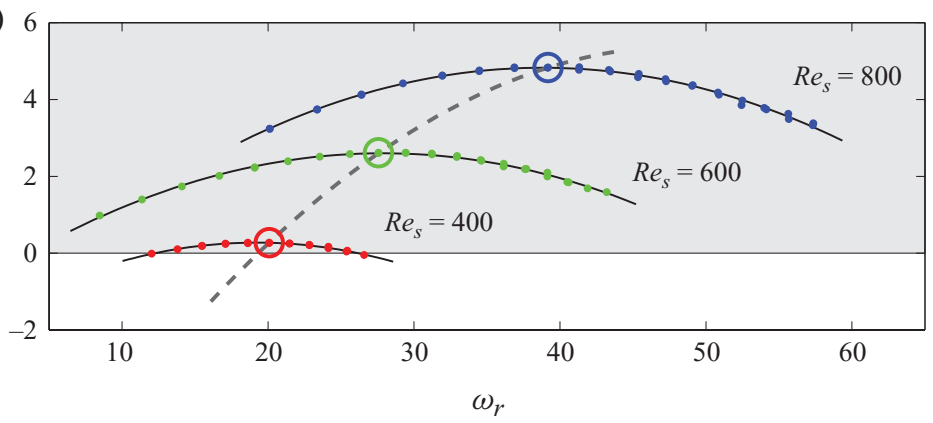

FIGURE 2. (a) Part of the full global spectrum showing the least-stable boundary-layer modes (in blue) and the slow-moving set of acoustic modes (in black) for $R e_{s}=800, M a_{s}=1.25$, $\theta_{w} \approx 1$ and $\beta=0.314$, as presented in Mack \& Schmid (2011); unstable half-plane shown by light grey. (b) Least-stable eigenvalues, belonging to global boundary-layer modes, of the global spectrum for selected sweep Reynolds numbers $\operatorname{Re}_{s}\left(M a_{s}=1.25, \theta_{w} \approx 1\right.$ and $\left.\beta=0.224\right)$. The dashed line indicates the evolution of the maximum growth rate.

boundary condition. For more details about the DNS-based global stability solver, the reader is referred to Mack \& Schmid (2010b).

\section{Results}

Compressible flow around a swept parabolic body (see figure 1) exhibits a complex perturbation dynamics and thus features a wide and rich variety of global modes. This fact has already been discussed in Mack \& Schmid (2011), where several types of global modes, such as stable and unstable boundary-layer modes, stable and unstable acoustic modes and stable wavepacket modes, have been uncovered for $R e_{s}=800, R e_{R}=129136, M a_{s}=1.25, \theta_{w} \approx 1$ and $\beta=0.314$. Furthermore, the present flow configuration is governed by a large number of parameters describing various flow quantities, fluid properties and geometric characteristics of the flow. Among these parameters, the influence of the spanwise wavenumber $\beta$ and the leading-edge Reynolds number $R e_{R}$ on the global stability of the flow has been briefly addressed in Mack \& Schmid (2011). A more comprehensive study including the neutral curve will be presented in what follows.

\subsection{Spectrum and global modes}

Boundary-layer instabilities have been found in previous studies (Mack \& Schmid 2011) to play a dominant role in the transition process. For this reason, we start by presenting results from the global spectrum which is displayed in figure 2(a) for a sweep Reynolds number of $R e_{s}=800$ and a spanwise wavenumber of $\beta=0.314$. It shows (in blue) a characteristic parabolic shape of discrete global modes which are unstable for a frequency range of $36 \lesssim \omega_{r} \lesssim 93$. The most unstable modes from this branch for $R e_{s}=800,600$ and 400 are shown in figure $2(b)$; this time the spanwise disturbance wavenumber was chosen as $\beta=0.224$ which is close to 0.213 , the value for the most amplified perturbations (see Mack \& Schmid 2011). Figure 2(b) further shows that decreasing $R e_{s}-$ which is equivalent to decreasing the leading-edge Reynolds number $R e_{R}$ and thus the leading-edge radius $R$ (see table 1) - has a stabilizing effect on boundary-layer modes.

In figure 3, we present the spatial distribution of three global boundary-layer modes, each belonging to the most unstable eigenvalue (depicted by a circle) of the three 


\begin{tabular}{lccc}
$R e_{s}$ & \multicolumn{1}{c}{$\operatorname{Re}_{R}$} & $\delta$ in $10^{-4}(\mathrm{~m})$ & $\bar{R}^{*}$ \\
800 & 129136 & 1.968 & 840 \\
600 & 72639 & 2.624 & 630 \\
400 & 32284 & 3.935 & 420
\end{tabular}

TABLE 1. Values of the Reynolds numbers, as defined in $\S 2$, of the investigated parameter choices.
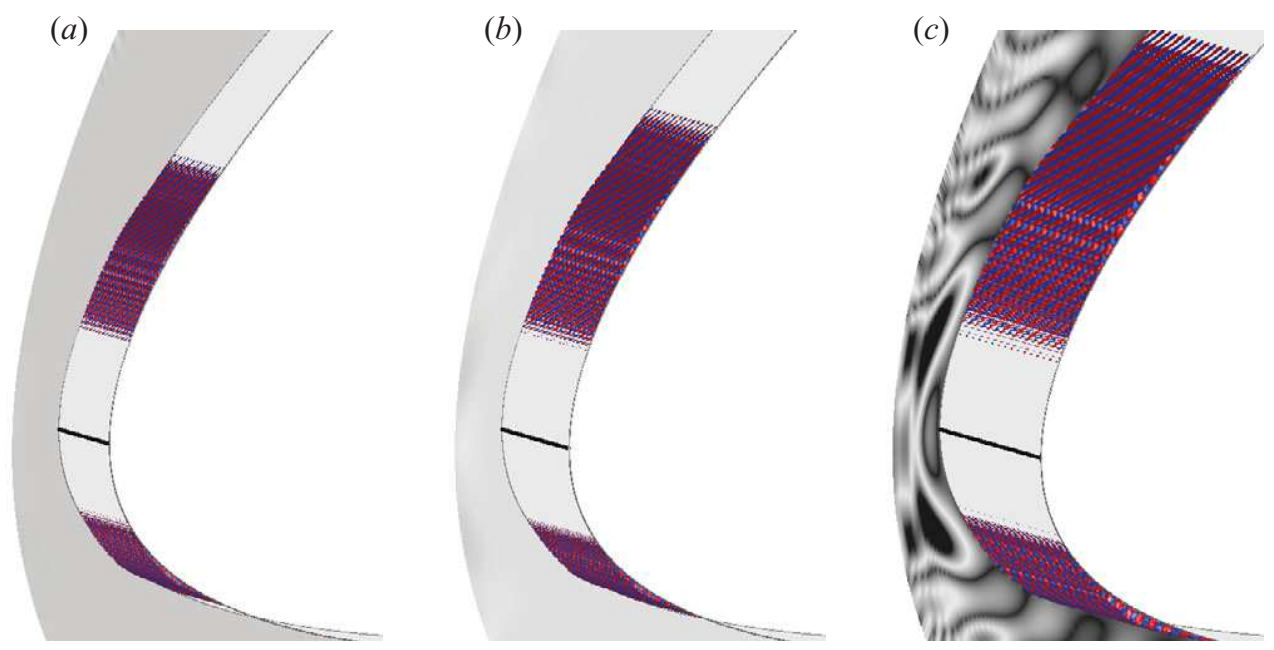

FiguRE 3. Spatial structure of the most unstable boundary-layer modes (depicted by circles in figure $2 b$ ) visualized by iso-surfaces (positive values in red and negative values in blue) of the normal velocity $v(x, y, z)=\operatorname{Re}\{\widetilde{v}(x, y)(\cos \beta z+\mathrm{i} \sin \beta z)\}$, where $\mathrm{Re}$ is real part; eight periods in $z$ are shown; attachment line in black: (a) $\operatorname{Re}_{s}=800$, (b) $\operatorname{Re}_{s}=600$ and (c) $\operatorname{Re}_{s}=400$. Contours of the associated pressure field of each mode are displayed in the background. Based on the chosen colour map for the pressure, only the acoustic footprint of the global boundary-layer mode for $R e_{s}=400$ is visible; the associated acoustic components for $R e_{s}=600$ and 800 are too weak to appear in the visualizations.

eigenvalue branches in figure $2(b)$. The modes are visualized by iso-surfaces of the normal velocity $v$, and eight periods in the spanwise $z$-direction are used; furthermore, contours of the associated pressure field of each mode are plotted in a wall-normal cross-plane at $z=0$, due to the chosen colour map the pressure distribution of the boundary-layer modes is only visible in figure 3(c), though. The noticeably different size of the domain in $z$, for a fixed wavenumber $\beta$, is the consequence of our definition of the spanwise length scale $L_{z}=(2 \pi / \beta) \delta$, where the viscous length scale $\delta$ depends on the sweep Reynolds number $\operatorname{Re}_{s}$ (see table 1). The disturbance wavelength thus scales with the length scale $\delta$ and the thickness of the boundary layer $\delta_{99} \approx 2.38 \delta$. Regarding the structure of the global modes in figure 3, we identify typical features of crossflow instabilities: co-rotating vortices that almost align with the external streamlines. Closer inspection of their spatial distribution, as $R e_{s}$ is decreased from 800 to 400 (see figure $3 a-c$ ), reveals, as we proceed downstream from the attachment line, (i) a weaker spatial exponential growth, causing (ii) a shift of the maximum amplitude in the chordwise direction, followed by (iii) a weaker exponential decay further downstream in the chordwise direction. A consequence of this behaviour is, on the one hand, a more compact spatial distribution closer to the attachment 
line of more unstable global modes at higher Reynolds numbers and, on the other hand, a substantially more elongated spatial distribution farther downstream from the attachment line of less unstable global modes. It is these latter modes that are capable of describing the dynamics in the entire leading-edge region.

Regarding the associated pressure field of the global modes in figure 3, an interesting feature is observed. While the global modes in figure $3(a, b)$ do not display any pronounced pressure field, the global mode in figure $3(c)$ adopts a pressure distribution which is known from the least-stable acoustic mode of the slow-moving acoustic modes (see figure $2 a$, marked by a black circle). This is a consequence of the coalescence of two eigenvalues, the least-stable eigenvalues of the respective types, for the particular parameter choice $R e_{s}=400$ and $\beta=0.224$, which results in a composite mode (showing typical features of boundary-layer modes and the least-stable acoustic mode). Noticeable grid effects in figure 3 demonstrate that the limit of numerical resolution of the fine spatial features of the global modes has been reached; careful studies have however shown that the least stable part of the global spectrum and the neutral curve are grid-independent and converged for the chosen numerical parameters.

The global nature of our geometry makes a direct comparison with local stability results rather difficult. A comprehensive study - morphing a complex geometry gradually into a simpler one while tracing the global spectrum as it approaches a superposition of familiar local spectra - would be desirable, but is prohibitively expensive for our configuration. The global modes, as they pertain to the neutral stability curve, will thus be categorized by their individual temporal dynamics and spatial features. Stationary boundary-layer modes - commonly observed in a local setting - have not been found for our configuration and parameter settings, despite efforts to focus on the $\omega_{r}=0$-axis by appropriately choosing the shift parameter $\sigma$ in the Cayley transformation.

\subsection{Neutral curve}

The neutral curve for supersonic flow about a swept parabolic body is presented in figure 4. In this figure, we display contours of constant growth rate $\omega_{i}$ for the boundary-layer modes (red) and the acoustic modes (blue). The presence of unstable global modes of boundary-layer as well as acoustic type yields a composite neutral stability curve delineating parameter regimes across which either boundary-layer or acoustic modes change from stable to unstable. Boundary-layer instabilities prevail for small spanwise wavenumbers $\beta$ and cease to exist below a critical Reynolds number of $R e_{s, \text { crit }} \approx 375$ (for $\beta=0.213$ ). For rather large values of the spanwise disturbance wavenumber $\beta$ acoustic instabilities dominate the linear stability of the flow even for sweep Reynolds numbers $R e_{s, c r i t}<375$. However, for $R e_{s}<170$ acoustic instabilities cease to exist as well.

For more detailed information, cross-cut profiles of the neutral curve for selected values of $R e_{s}$ are presented in figure 5. The cross-cuts (as well as the neutral curve) show that for large values of $R e_{s}$ the boundary-layer modes (denoted by the crossflow branch) exhibit significantly larger growth rates $\omega_{i}$ than the acoustic modes. These growth rates decay linearly with $R e_{s}$; the maximum growth rate is always found at a critical spanwise wavenumber of $\beta=0.213$. The acoustic modes, on the other hand, show rather weak growth rates, do not scale linearly with $R e_{s}$ and do not show a Reynolds-number-independent critical value of $\beta$.

The neutral curve displayed in figure 4 represents a two-dimensional cut through a high-dimensional parameter space. The immediate choice of varying parameters consists of the spanwise wavenumber $\beta$ (based on a one-time computed base flow) 


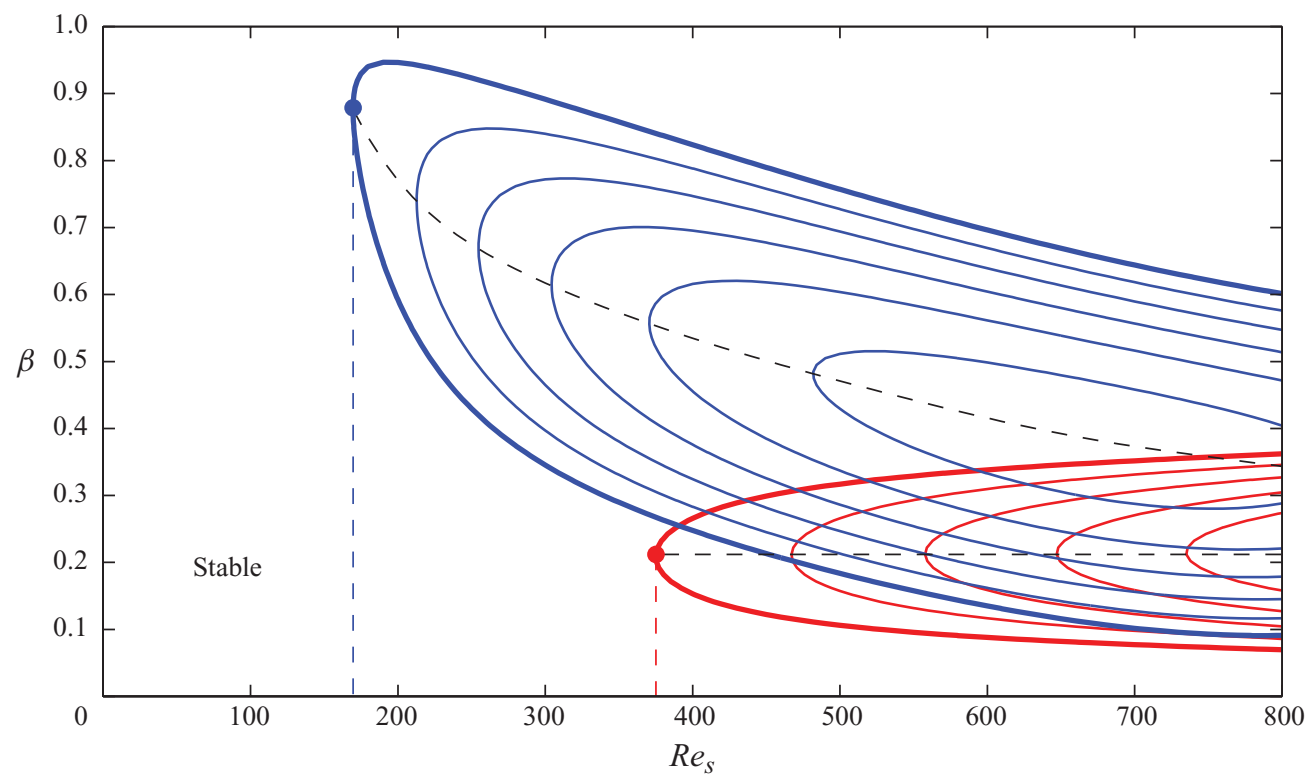

FigURE 4. Neutral curve for supersonic flow about a swept parabolic body for a sweep Mach number $M a_{s}=1.25$ and an adiabatic wall (boundary-layer modes in red and acoustic modes in blue); contour spacing for the boundary-layer modes $\Delta \omega_{i}=1$, contour spacing for the acoustic modes $\Delta \omega_{i}=0.1$. The dashed lines indicate the evolution of the maximum value of the growth rate.
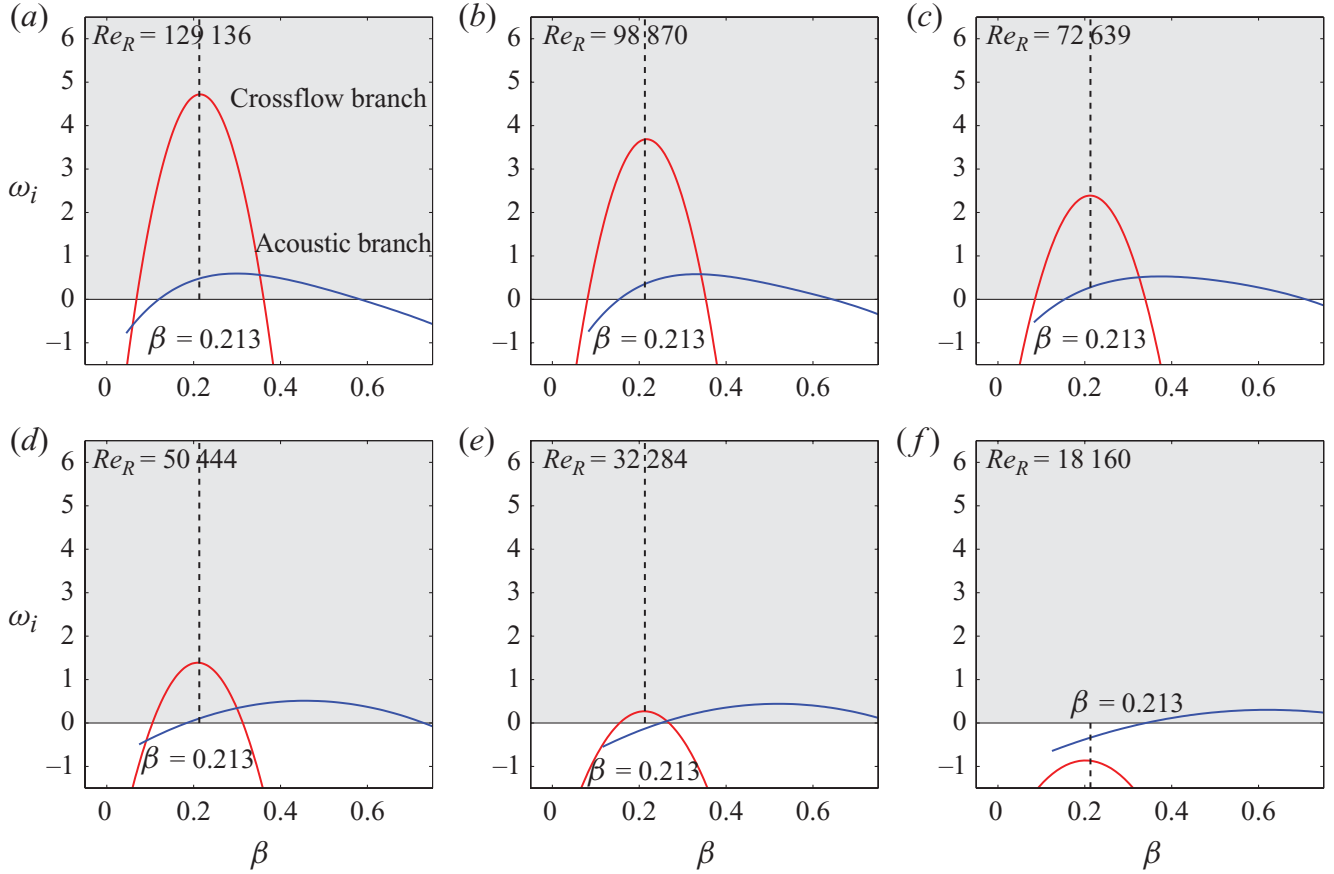

Figure 5. Cross-cuts of the neutral curve in figure 4 showing the influence of $\beta$ on $\omega_{i}$ for selected values of $R e_{s}$ : (a) $R e_{s}=800$, (b) $R e_{s}=700$, (c) $R e_{s}=600$, (d) $R e_{s}=500$, (e) $R e_{s}=400$ and $(f) R e_{s}=300$. The unstable half-plane is visualized in light grey. 
and the sweep Reynolds number $R e_{s}$ (requiring the computation of a new base flow for each value of $R e_{s}$ ). While for these two parameter studies the free-stream conditions remain unchanged, investigating the influence of additional parameters (sweep angle, sweep Mach number, wall temperature ratio, etc.) requires a substantial effort owing to the added complication of a nonlinear dependence of these parameters on the free-stream conditions (see Mack \& Schmid 2011 for details).

\section{Discussion and conclusions}

The stability of supersonic flow around a swept parabolic body has been studied using a global approach based on an inclusive flow model which incorporates attachment-line instabilities and crossflow vortices. These two instabilities have been identified as the dominant mechanisms for the onset of transition in swept leadingedge boundary-layer flow. Global boundary-layer and acoustic modes have been found to become unstable for specific parameter combinations, and a neutral stability curve for these two types of modes has been presented as a function of the spanwise disturbance wavenumber and the sweep Reynolds number. Two critical Reynolds numbers, based on a boundary-layer or an acoustic instability, have been determined.

The instability mechanism based on boundary-layer modes follows the classical pattern of shear-induced instabilities of generic wall-bounded shear layers, i.e. the amplification of disturbances by extraction of energy from the base flow. The instability mechanism underlying the acoustic branch of the neutral curve stems from the parametrically resonant amplification of sound waves as they are reflected between the bow shock and the rigid wall. The geometry of the bow shock and the wall, as well as their respective reflective behaviour for acoustic wave, influences the overall amplification potential of acoustically driven instabilities: a weak instability can be observed for a significant range of parameter values. Examples of the spatial shape of unstable modes from the acoustic branch are given in Mack \& Schmid (2011). To our knowledge, these types of instabilities have not been observed experimentally; only the influence of compressibility on the boundary layer modes has been discussed (see Gaillard et al. 1999).

Central to our analysis is the global stability approach and consequently the realization that the computed global modes are of composite type combining features from boundary-layer and acoustic instabilities. Even though the global boundarylayer modes show characteristics that are known from the stability analysis of local flow models (for the attachment-line and the flow further downstream), our approach treats the coherent dynamic structures of the flow as one entity. Consequently, stability information obtained from a local approach, which constitutes our current theoretical understanding of swept leading-edge flow, is expected to differ from results based on a global approach.

A stability analysis based on a global point of view - avoiding the limitations of local flow models - constitutes a novel approach for swept leading-edge flow, promises new insight into the inherent instability mechanisms and has the potential to uncover a wealth of stability behaviour as stated by Joslin (1996). The computed global eigenmodes have been found to be non-orthogonal and, consequently, transient effects are expected to play a significant role in the description of disturbance and bifurcation behaviour near the critical parameter values. In particular, for the subcritical regime the global stability approach taken in this article is expected to give new results which will help answer some of the remaining questions regarding the onset of transition for swept leading-edge flow. 
Financial support from the Deutsche Forschungsgemeinschaft (DFG), the Studienstiftung des Deutschen Volkes, the Alexander-von-Humboldt Foundation and the ANR programme 'Chaires d'excellence' is gratefully acknowledged.

\section{REFERENCES}

Gaillard, L., Benard, E. \& Alziary de Roquefort, T. 1999 Smooth leading-edge transition in hypersonic flow. Exp. Fluids 26, 169-176.

GASTER, M. 1965 A simple device for preventing turbulent contamination on swept leading edges. J. R. Aeronaut. Soc. 69 (659), 788-789.

Gaster, M. 1967 On the flow along swept leading edges. Aeronaut. Q. 18 (2), 165-184.

GraY, W. E. 1952 The effect of wing sweep on laminar flow. Tech. Rep. RAE TM Aero 255. British Royal Aircraft Establishment.

Hall, P. \& MaliK, M. 1986 On the instability of a three-dimensional attachment-line boundary layer: weakly nonlinear theory and a numerical approach. J. Fluid Mech. 163, 257-282.

Hall, P., Malik, M. \& Poll, D. I. A. 1984 On the stability of an infinite swept attachment-line boundary layer. Proc. R. Soc. Lond. A 395, 229-245.

JosLin, R. D. 1995 Direct simulation of evolution and control of three-dimensional instabilities in attachment-line boundary layers. J. Fluid Mech. 291, 369-392.

JosLIN, R. D. 1996 Simulation of three-dimensional symmetric and asymmetric instabilities in attachment-line boundary layers. AIAA J. 34 (11), 2432-2434.

LehoucQ, R. B., Sorensen, D. C. \& YAng, C. 1998 ARPACK Users' Guide: Solution of Large-Scale Eigenvalue Problems with Implicitly Restarted Arnoldi Methods. SIAM.

LiN, R. S. \& MALIK, M. R. 1996 On the stability of attachment-line boundary layers. Part 1. The incompressible swept Hiemenz flow. J. Fluid Mech. 311, 239-255.

Mack, C. J. \& Schmid, P. J. $2010 a$ Direct numerical study of hypersonic flow about a swept parabolic body. Comput. Fluids 39, 1932-1943.

Mack, C. J. \& Schmid, P. J. $2010 b$ A preconditioned Krylov technique for global hydrodynamic stability analysis of large-scale compressible flows. J. Comput. Phys. 229 (3), 541-560.

MACK, C. J. \& SCHMID, P. J. 2011 Global stability of swept flow around a parabolic body: features of the global spectrum. J. Fluid Mech. 669, 375-396.

MAck, C. J., Schmid, P. J. \& SesterhenN, J. S. 2008 Global stability of swept flow around a parabolic body: connecting attachment-line and crossflow modes. J. Fluid Mech. 611, 205-214.

Pfenninger, W. 1965 Some results from the X-21 program. Part I. Flow phenomena at the leading edge of swept wings. Tech. Rep. 97. AGARDograph.

Poll, D. I. A. 1979 Transition in the infinite swept attachment-line boundary layer. Aeronaut. $Q$. 30, 607-628.

PoLL, D. I. A. 1983 The development of intermittent turbulence on a swept attachment line including the effects of compressibility. Aeronaut. Q. 34, 1-23.

Poll, D. I. A. 1984 Transition description and prediction in three-dimensional flows. In Special Course on Stability and Transition of Laminar Flow, pp. 5/1-5/23. AGARD.

Semisynov, A. I., Fedorov, A. V., Novikov, V. E., Semionov, N. V. \& Kosinov, A. D. 2003 Stability and transition on a swept cylinder in a supersonic flow. J. Appl. Mech. Tech. Phys. 44, 212-220.

SpalaRT, P. R. 1988 Direct numerical study of leading-edge contamination. In AGARD-CP-438, pp. $5 / 1-5 / 13$. 